\title{
Catalogue of HI maps of galaxies. I. ${ }^{\star}$
}

\author{
M.C. Martín \\ Instituto Argentino de Radioastronomía (IAR), Casilla de Correo 5 Villa Elisa (1894), Provincia de Bs. As., Argentina
}

Received August 1, 1995; accepted December 11, 1997

\begin{abstract}
A catalogue is presented of galaxies having large-scale observations in the HI line. This catalogue collects from the literature the information that characterizes the observations in the $21-\mathrm{cm}$ line and the way that these data were presented by means of maps, graphics and tables, for showing the distribution and kinematics of the gas. It contains furthermore a measure of the HI extension that is detected at the level of the maximum sensitivity reached in the observations. This catalogue is intended as a guide for references on the HI maps published in the literature from 1953 to 1995 and is the basis for the analysis of the data presented in Paper II.
\end{abstract}

Key words: catalogs - galaxies: ISM; fundamental parameters — radio lines: galaxies

\section{Introduction}

The Magellanic Clouds were the first extragalactic objects observed and mapped in the 21-cm line (Kerr \& Hindman 1953; Kerr et al. 1954). Soon, the large angular extension of some nearby objects enabled one to obtain other detailed maps of the HI gas. At present, the density distribution of neutral hydrogen is known for a large number of galaxies.

Since neutral hydrogen is typically found farther out in the disk than optical components, it constitutes an important tool in the study of the dynamics of a galaxy. With respect to the morphology, some high-resolution studies were able of distinguish structures like spiral arms, bars, bubbles, holes of HI, etc. In some galaxies, gas appendages are often seen to extend outward from the disks, like "plumes", "tails", connecting "bridges" and other such features. Some of these appendages have been explained

Send offprint requests to: M.C. Martín

* The catalogue is only available in electronic form at the CDS via anonymous ftp 130.79 .128 .5 or http://cdsweb.ustrasbg.fr/Abstract.html in terms of tidal interactions amongst neighbouring galaxies, but not all. Other studies have shown that some galaxies exhibit enormously extended HI disks, with important implications. The use of the interstellar gas as a tracer of the gravitational potential produced by the total content of the galaxy, by means of rotation curves or velocity fields, is dynamically important. Only if we know more details of the structure and distribution of the different components of a galaxy, particularly the HI gas, have we an insight into the question of galaxy evolution.

Our initial purpose was to study the gas distribution in the galaxies, to find any possible connection between the extension of the HI gas and other properties of the galaxies (morphological type, size, surface brightness, etc.) using a large sample. To achieve this, we began by taking into account those papers in which it was possible to consider a gaussian distribution for a simpler analysis, as is shown in Paper II. However, during the bibliographical search, we found in the literature many papers related to studies of the HI distribution in galaxies, most of which were not useful for our initial purpose. Nevertheless, and because of the lack of any catalogue providing information about large-scale HI observations in extragalactic systems, we considered that it would be interesting to collect the references, to provide astronomers a guide to the HI maps of galaxies published up to 1995 .

The maps collected for this catalogue are described in Sect. 2. The information extracted from the literature and the measurements made on the maps are presented in Sect. 3. Finally, the data are briefly discussed in Sect. 4.

\section{Collection of the maps}

The body of HI data on a galaxy can be thought of as an array of intensities in a three dimensional system: two angular coordinates in the sky plane and radial velocity. This array is frequently referred to as a "data cube". The array values intend to show the distribution and kinematics of the gas and are displayed by means of two-dimensional maps, graphics and tables, which will be referred to from 
now on simply as maps. We have catalogued the maps that are representative of the overall distribution of gas. Those maps referred to particular structures in a galaxy (HI holes, bubbles, shells, tails, etc.) are only mentioned in the notes. The type of maps included in the catalogue are:

a) The distribution of the total HI emission integrated over all radial velocities.

b) Channel maps, or maps of intensities at a given velocity.

c) Position-velocity diagrams, or maps of the intensity as a function of velocity and one of the coordinates, the other being kept fixed.

d) HI velocity field, or maps of some kind of velocity derived from the spectra (peak, median, etc.) as a function of position.

e) Rotation curves derived from the HI data.

f) Variation of some parameters along one coordinate. This type of map may be drift scans (variation of temperature with the position at a given velocity) or distribution of the HI emission (as line integral, surface density, HI fluxes, etc.) along one coordinate on the galaxy.

g) HI profiles at different positions inside or near the optical galaxy.

h) Global HI profile obtained by adding the large-scale observations.

i) Tables of data of the large-scale observations.

Other types of maps are put in the footnotes, such as dispersion velocity maps, peak brightness distribution, etc.

For this catalogue, we have applied the following selection criteria to reach a somewhat complete sample: We have taken into account only references in main journals, and we have included even galaxies with few observed positions (two or three) which can give a crude distribution of the gas. Sometimes, we found the same HI maps published in an IAU Symposium as well as in a major journal, as in the case of NGC 4038/39 (van der Hulst 1978 and 1979), or M 33 (Davies 1972 and de Jager \& Davies 1971). In these cases, we took the reference that contains the main work. Other times, we found two or more references related to the same observational data. In these cases, we took the observational paper, unless new maps (with different resolution or sensitivity) were presented with the same data, e.g. NGC 6946: Carignan et al. (1990) and Boulanger \& Viallefond (1992). Furthermore, preliminary reports on the observations, published in an IAU Symposium or a short letter, are only mentioned in the footnotes of the catalogue.

\section{The catalogue: Parameters and measurements from the maps}

For each galaxy, the following information was collected for constructing the catalogue:
1. Optical parameters of the galaxy: name; coordinates; corrected diameter and morphological type, extracted from LEDA (Lyon-Meudon Extragalactic Database, first and second edition). For uncatalogued galaxies, the name and coordinates were taken as given by the authors or were estimated from the maps.

2. HI observational parameters: the telescope used with its angular resolution; the velocity resolution of the map and the achieved sensitivity represented by the rms noise in the maps.

3. The type of map, as described above and codified as described in the Read.me file.

4. The value of the lowest contour level of the gas distribution, only for types of maps as described in 2-a). This contour level usually is related to the sensitivity, because it is, in general, 2, 3 or 4 times the rms noise of the observation. The usual unit of contour level is the column density $\left(n_{\mathrm{HI}}\right)$ expressed in atoms $\mathrm{cm}^{-2}$ (assuming optically thin gas), and this is the one used in this catalogue. When the levels are expressed in other units without any possibility of changing them into the $n_{\mathrm{HI}}$ values (e.g., when antenna temperatures or flux densities are used instead of brightness temperature), the value is lost and the condition is explained in a note. It is worth noticing that these surface densities are always beam-averaged, because in some way this effect will influence the HI diameter described in the next item.

5. A measure of the HI extension spread by the gas, determined by the lowest isophote quoted in the previous item. These measurements were made by us and only for the type of maps described in 2-a). The outer regions of a disk are very fragile and can be severely affected by a violent environment. Since for statistical purposes, it is more convenient to deal with relatively unperturbed systems, the measurement of the extension intends to embrace the undisturbed gas. So it does not take into account those structures that probably have a tidal origin, like tails, plumes, HI streams and bridges extending outside the galaxy. Sometimes, however, it is not possible to distinguish these structures from the maps due to beam smearing, and then they are inevitably included in the measure of the HI extension. An example of this is NGC 4038/39, whose tail is resolved by van der Hulst (1979), but not by Rots (1980).

Some maps show filamentary structures or HI clumps or "patchy structure" extending outside the general distribution of the galaxy. The HI extension does not include them, unless these structures are undoubtedly associated with the galaxy.

Finally, the measure of the gas extension has not, by itself, any sense unless it is referred to a sensitivity level. Therefore, when we were not able to find the $n_{\mathrm{HI}}$ value, the extension of the gas is not given. 
With respect to the errors of these measurements, they come basically from two different sources: from the author of this catalogue and from the literature. That is, there are errors inherent to the measurements and possible errors in the maps published in the literature. For the first type of error, a favourable point is the fact that the measurements were made by only one person, who applied uniform criteria to do them. Whenever there was a doubt concerning the measurement (e.g. when the lower contour level is not well-defined or has distortions, plumes, warps, etc., or the outlying gas vanishes in a clumpy structure), we express it with the $\sim$ symbol. For the second type of error, we searched the literature for possible errata of the works.

6. The hydrogen mass, as given in the reference and by integration of the HI map. This quantity was unaffected by distance effects, to be helpful in the comparison of the total gas emission collected by different telescopes.

7. Footnotes that remark: HI appendages such as tails, plumes or bridges; references of works related to particular structures in a galaxy, such as $\mathrm{HI}$ holes or shells (particularly in nearby galaxies); lost entry parameters; references for errata; etc. These notes have the purpose of improving this catalogue.

Sometimes, we can see from the maps that two or more galaxies have a common envelope of gas. This envelope may be due to a genuine bridge of material joining the galaxies, or it may be due to the beam smearing; that is, it is not possible to remove the individual gas structures of the galaxies due to the low spatial resolution of the telescope. Other times, the author considers that more than one galaxy contributes to the gas emission. In these cases, the galaxies involved in the same HI structure are marked, and the parameters mentioned in previous items (1 to 7 ) correspond to all galaxies, as a whole.

\section{Comments on the catalogue}

This catalogue contains information for about 1400 galaxies, with more than 500 references.

For the first twenty years, single-dish telescopes provided the vast majority of the HI data. As can be seen in the catalogue, most of the observations were provided by Green Bank 91-m, Jodrell Bank, Nançay, Effelsberg, Parkes and Arecibo telescopes. Later, with the requirement of higher resolution, the techniques of spectral line aperture synthesis appeared, developed especially at Cambridge, Green Bank, Owens Valley, Westerbork, NRAO (VLA) and more recently, in Australia (ATCA). With these large telescopes, particular structures such as holes, bubbles, HI concentrations in spiral arms, etc., started to be resolved. More galaxies seem to exhibit large extensions of gas, such as plumes or long tails, that cannot be classified as bridges, because there are no obvious optical companions. With the high spatial resolution of these large telescopes, HI images of uncatalogued small galaxies have also appeared in the observational fields. Suitable deep optical images and/or redshifts are needed to confirm the existence of these galaxies.

Some galaxies have several observations made by different authors and at telescopes. In these cases, we can see a general increase of the $\mathrm{HI}$ extension as the sensitivity increases. The extension of gas undoubtedly depends on the sensitivity reached in the observation. When dealing with large extensions of gas, it is necessary to specify the sensitivity level. There is also an apparent increase of the gas extension as the resolution of the telescope decreases. In any case, despite the dependence of the gas extension on the telescope beam and on the observational sensitivity level, a first glance at the catalogue shows that the neutral hydrogen gas is typically detected farther out than the optical component. The exceptions are clear for the galaxies of the Virgo Cluster NGC 4438, NGC 4569 and NGC 4388 which, beyond any doubt, have gas deficiencies.

Acknowledgements. We are grateful to the referee, Dr. W.K. Huchmeier, for valuable suggestions. I also appreciate important remarks on the text by Dr. C. Olano. Thanks also to Dr. E. Bajaja for useful comments on the original manuscript.

\section{References}

Boulanger F., Viallefond F., 1992, A\&A 266, 37

Carignan C., Charbonneau P., Boulanger F., Viallefond F., 1990, A\&A 234, 43

Davies R.D., 1972, IAU Symp. 44, 67

de Jager G., Davies R.D., 1971, MNRAS 153, 9

Kerr F.J., Hindman J.V., 1953, AJ 56, 218

Kerr F.J., Hindman J.V., Robinson B.J., 1954, Aust. J. Phys. 7, 297

Rots A.H., 1980, A\&AS 41, 189

van der Hulst J.M., 1978, IAU Symp. 77, 269

van der Hulst J.M., 1979, A\&A 71, 131 